\title{
Infecciones espontáneas en el paciente cirrótico: características clínicas y microbiológicas en una cohorte bi- institucional del Uruguay.
}

Martín Elizondo ORCID: 0000-0001-8396-6588 Médico. Internista. Unidad BiInstitucional de Trasplante Hepático

- UDA CeNaHBP - Servicio de Enfermedades Hepáticas HCFFAA.

Bruno Mirza

ORCID: 0000-0002-3107-4104 Asistente Interino de Clínica Médica

"A" - Hospital de Clínicas "Dr. Manuel Quintela" FMed - UdelaR.

María Noel García ORCID: 0000-0002-0786-3568

Residente de Medicina Interna.

Clínica Médica "2" - Hospital

Pasteur. Rotante de la Unidad BiInstitucional de Trasplante Hepático

- UDA CeNaHBP - Servicio de

Enfermedades Hepáticas HCFFAA.

Marcelo Valverde ORCID: 0000-0002-4592-0762

Especialista en Medicina Interna, Emergentología y Hepatología de Adulto. Prof. Adj. Clínica Médica "A" - Hospital de Clínicas "Dr. Manue Quintela" FMed - UdelaR. Unidad BiInstitucional de Trasplante Hepático

- UDA CeNaHBP - Servicio de Enfermedades Hepáticas HCFFAA.

Solange Gerona ORCID: 0000-0003-0771-180

Especialista en Gastroenterología y Hepatología del Adulto. Fellow de la AASLD. Jefa de la Unidad BiInstitucional de Trasplante Hepático

- UDA CeNaHBP - Servicio de Enfermedades Hepáticas HCFFAA.

Gabriela Ormaechea ORCID: 0000-0002-2981-7722 Especialista en Medicina Interna. Fellow de la ACP. Prof. Dir. Clínica Médica "A" - Hospital de Clínicas "Dr. Manuel Quintela" FMed UdelaR

\section{Spontaneous infections in cirrhotic patients: clinical and microbiological characteristics in a bi-institutional cohort from Uruguay. \\ Infecções espontâneas em pacientes cirróticos: características clínicas e microbiológicas em uma coorte biinstitucional do Uruguai.}

Resumen: Los pacientes cirróticos hospitalizados presentan una alta prevalencia de infecciones bacterianas. Entre ellas, las denominadas infecciones espontáneas del cirrótico, que incluyen la peritonitis bacteriana espontánea, la bacteriemia espontánea y el empiema bacteriano espontáneo, constituyen un grupo particular, con un elevado riesgo de complicaciones y mortalidad. El presente trabajo tiene como objetivo describir y caracterizar las infecciones espontáneas en el cirrótico, la microbiología prevalente y su perfil de resistencia antibiótica, en 2 centros asistenciales de Montevideo-Uruguay. Se realizó un estudio transversal, descriptivo y multicéntrico, que incluyó pacientes con cirrosis hepática hospitalizados, en el periodo marzo 2018-marzo 2019. Del total de pacientes ingresados con cirrosis (156), 25 (16\%) desarrollaron una infección espontánea del cirrótico. En 24 de estos pacientes (96\%) la cirrosis se encontraba en estadios avanzados (Child-Pugh B-C, MELD >15). La forma de presentación más frecuente fue la peritonitis bacteriana espontánea (52\%), seguida de la bacteriemia espontánea (40\%) y del empiema bacteriano espontáneo (8\%). El aislamiento microbiológico fue posible en 17 casos (68\%). En 12 de ellos (70\%) se aislaron bacilos Gram negativos, en 4 (24\%) bacilos Gram positivos, y Candida en 1 solo caso. Se identificaron 3 cepas productoras de BLEE (2 K. pneumoniae y $1 \mathrm{E}$. coli), y 4 microorganismos con otros perfiles de resistencia antibiótica (2 E. faecalis, $1 \mathrm{~K}$. oxytoca, $1 \mathrm{E}$. cloacae). La prevalencia de microorganismos multirresistentes fue del $41 \%$, siendo todos los casos en el grupo de adquisición nosocomial. La realización de otros estudios en esta área podría aproximarnos al conocimiento de la epidemiología local, para la optimización de los tratamientos empíricos dirigidos a los microorganismos más prevalentes, con el objetivo de mejorar el abordaje de las infecciones en los pacientes con cirrosis.

Palabras clave: Peritonitis bacteriana espontánea, bacteriemia espontánea, empiema bacteriano espontáneo, cirrosis.

Abstract: Hospitalized cirrhotic patients present with a high prevalence of bacterial infections. Among them, spontaneous cirrhotic infections include spontaneous bacterial peritonitis, spontaneous bacteremia and spontaneous bacterial empyema, which constitute a particular group, with a high risk of complications and mortality. The work above aims to describe and characterize the spontaneous infections in the cirrhotic, the prevalent microbiology and its resistance profile, in 2 healthcare centers in Montevideo-Uruguay. A multicenter, descriptive, cross-sectional study was held, including hospitalized patients with liver cirrhosis, between March 2018 - March 2019. 25 (16\%) of the 156 patients admitted with cirrhosis developed a spontaneous cirrhotic infection. 24 (96\%) of these patients were in an advanced stage of their disease, (Child-Pugh B-C, MELD>15). The most frequent presentation was the spontaneous bacterial peritonitis (52\%), followed by the spontaneous bacteremia $(40 \%)$ and the spontaneous bacterial empyema (8\%). Microbiological isolation was possible in 17 cases (68\%), of which 12 (70\%) developed gram negative bacilli, 4 (24\%) gram positive bacilli and 1 case Candida. 3 ESBL 
producing strains were identified, $(2 \mathrm{~K}$. pneumoniae and $1 \mathrm{E}$. coli), and other 4 microorganisms with different antibiotic resistance profile ( $2 \mathrm{E}$. faecalis, $1 \mathrm{~K}$. oxytoca, $1 \mathrm{E}$. cloacae). The prevalence of multiresistant microorganisms was $41 \%$, all of nosocomial acquisition. Research on this topic may provide more knowledge about the local epidemiology, improving the empiric treatments, targeting the most prevalent microorganisms, in order to better approach the infections in cirrhotic patients.

Key words: Spontaneous bacterial peritonitis, spontaneous bacteremia, spontaneous bacterial empyema, cirrhosis.

Resumo: Pacientes cirróticos hospitalizados presentam alta prevalência de infecções bacterianas. Dentre elas, as chamadas infecções cirróticas espontâneas, que incluem a peritonite bacteriana espontânea, a bacteremia espontânea e o empiema bacteriano espontâneo, constituem um grupo particular, com alto risco de complicações e mortalidade. O presente trabalho tem como objetivo descrever e caracterizar as infecções espontâneas em pacientes cirróticos, a microbiologia prevalente e seu perfil de resistência aos antibióticos, em 2 centros de saúde em Montevidéu-Uruguai. Foi realizado um estudo transversal, descritivo e multicêntrico, que incluiu pacientes hospitalizados com cirrose hepática, no período de março de 2018 a março de 2019. Do total de pacientes internados com cirrose (156), 25 (16\%) desenvolveram infecção cirrótica espontânea. A cirrose estava em estágios avançados, em 24 desses pacientes (96\%) (Child-Pugh B-C, MELD >15). A forma de apresentação mais frequente foi peritonite bacteriana espontânea (52\%), seguida de bacteremia espontânea (40\%) e empiema bacteriano espontâneo (8\%). O isolamento microbiológico foi possível em 17 casos (68\%). Em 12 deles (70\%) foram isolados bacilos Gram negativos, em 4 (24\%) bacilos Gram positivos e Candida em apenas 1 caso. Foram identificadas 3 cepas produtoras de ESBL (2 K. pneumoniae e $1 \mathrm{E}$. coli) e 4 microrganismos com outros perfis de resistência a antibióticos (2 E. faecalis, $1 \mathrm{~K}$. oxytoca, 1 E. cloacae). A prevalência de microrganismos multirresistentes foi de $41 \%$, todos no grupo de aquisição nosocomial. A realização de outros estudos nesta área poderá nos aproximar do conhecimento da epidemiologia local, para a otimização de tratamentos empíricos direcionados aos microrganismos mais prevalentes, com o objetivo de melhorar o manejo de infecções em pacientes com cirrose.

Palavras chave: Peritonite bacteriana espontânea, bacteremia espontânea, empiema bacteriano espontâneo, cirrose. 


\section{Introducción}

Las infecciones bacterianas son frecuentes en pacientes con cirrosis ${ }^{(1-3)}$, particularmente en aquellos que requieren internación, donde la prevalencia alcanza el $34 \%{ }^{(4)}$.

Dicha intercurrencia incrementa el riesgo de desarrollar complicaciones como injuria renal aguda $(30 \%)^{(5-9)}$, constituyendo una causa precipitante frecuente de falla hepática aguda sobre crónica, con un incremento de la mortalidad por 4 veces con relación a los pacientes cirróticos no infectados ${ }^{(10-12)}$.

Un grupo particular de infecciones son las denominadas infecciones espontáneas del cirrótico (IEC) que incluyen a la Peritonitis Bacteriana Espontánea (PBE), al Empiema Bacteriano Espontáneo (EBE) y la Bacteriemia Espontánea (BE), con particularidades en su patogenia y forma de presentación clínica ${ }^{(13)}$.

En el caso de las IEC la condición "sine qua non" es la existencia de translocación bacteriana (TB) desde la luz intestinal a los ganglios linfáticos mesentéricos.

Esta TB está relacionada con varios factores, incluyendo las características de la microbiota intestinal, la permeabilidad intestinal aumentada, y defectos, tanto locales como sistémicos, en la inmunidad del huésped ${ }^{(14)}$.

Respecto a la microbiota del paciente cirrótico cabe destacar que existe un sobrecrecimiento bacteriano como consecuencia de una disminución en la motilidad del intestino delgado por disfunción autonómica.

El aumento de la permeabilidad intestinal es consecuencia de múltiples factores: cambios ultra-estructurales en la mucosa intestinal, estrés oxidativo, inflamación local e hiperactividad del sistema nervioso autónomo.

En lo que respecta a la inmunidad del paciente cirrótico existen varias anormalidades de la respuesta inmunológica que conduce a un verdadero estado de inmunodeficiencia adquirida ${ }^{(15)}$.

Desde el punto de vista microbiológico, en los últimos años se ha reportado una creciente prevalencia de IEC ocasionadas por microorganismos multirresistentes (MOMR) y extremadamente resistentes, mostrando una prevalencia muy variada entre regiones, e incluso entre diferentes centros asistenciales dentro de una misma región geográfica (16-17).

Estas infecciones se asocian a peor pronóstico, generan dificultades en el manejo del paciente durante la internación, e incrementan los costos en salud ${ }^{(18-20)}$.

El presente trabajo surge a partir de dos centros asistenciales de nuestro país, con el objetivo general de describir y caracterizar las IEC (PBE, EBE y BE) que presentaron los pacientes con cirrosis hepática $(\mathrm{CH})$, al ingreso o durante la internación y los objetivos específicos de calcular la incidencia de IEC, describir la prevalencia de los microorganismos causantes de las IEC, determinar el perfil de resistencia antibiótica y caracterizar la severidad de la enfermedad hepática. Consideramos que será de utilidad para establecer estrategias de prevención y tratamiento de las mismas.

\section{Material y métodos}

Se trata de un estudio transversal, descriptivo y multicéntrico, que incluyó pacientes con diagnóstico de cirrosis hepática $(\mathrm{CH})$ que ingresaron en el Hospital de Clínicas (HC) y Hospital Central de las Fuerzas Armadas (HM), en el periodo comprendido entre marzo 2018 a marzo 2019, que presentaron una IEC al momento del ingreso o durante su estadía hospitalaria.

Los criterios de inclusión fueron pacientes mayores de 18 años con diagnóstico de $\mathrm{CH}$, que aceptaron participar del proyecto y que presentaron una IEC al ingreso o durante la internación.

Los criterios de exclusión fueron pacientes con otras condiciones que generen inmunodepresión como la presencia de virus de inmunodeficiencia humana, tratamiento inmunosupresor o tratamiento oncológico (quimioterapia).

El reclutamiento de los pacientes se realizó de forma programada con visitas regladas en los diferentes servicios de internación. Se solicitó el consentimiento informado escrito a todos aquellos pacientes que reunieron los criterios de inclusión y no presentaron criterios de exclusión para el presente estudio. Se realizó una revisión sistematizada de la historia clínica con el objetivo de obtener los datos que permitieran confirmar el diagnóstico de cirrosis, calcular los 
scores Child-Pugh, MELD-Na, y finalmente datos referentes a la/s IEC descritas (recuento de PMN del líquido de ascitis/hidrotórax, cultivos del/los mismo/s, hemocultivos). La recolección de datos fue volcada en una planilla de datos de Excel creada "ad-hoc" para su posterior análisis estadístico. El presente trabajo se realizó dentro de las normas vigentes de ética médica y fue aprobado por el Comité de Ética Médica de los Centros Hospitalarios participantes.

Se definieron las siguientes variables:

Cirrosis: demostración histológica, o por la suma de criterios bioquímicos, clínicos, endoscópicos y/o de métodos de imágenes.

Infecciones espontáneas del cirrótico (IEC): incluye: Peritonitis Bacteriana Espontánea (PBE), Bacteriemias Espontáneas (BE) y Empiema Bacteriano Espontáneo (EBE).

PBE: Recuento de polimorfonucleares $(P M N)>250 / m m 3$ en líquido ascítico, sin evidencia de fuente infecciosa intra-abdominal, quirúrgica o médica (con hemocultivos negativos o positivos) (14).

BE: hemocultivos positivos en ausencia de otra causa de bacteriemia, excluyendo gérmenes contaminantes. criterios:

EBE: infección de un hidrotórax hepático (o ascitis torácica), establecida según los siguientes

- Cultivo de líquido pleural positivo y recuento de PMN > 250 células/ $\mu \mathrm{L}$. Si el cultivo es negativo se requiere un recuento de $\mathrm{PMN}>500$ células $/ \mu \mathrm{L}$.

- Exclusión de derrame pleural paraneumónico/empiema pleural. ${ }^{(16)}$

Los datos se analizaron utilizando distribución de frecuencias para las variables cualitativas y medidas de resumen para las cuantitativas. La incidencia de IEC se calculó en base a la población total de pacientes con $\mathrm{CH}$ ingresados en el período de reclutamiento mencionado.

\section{Resultados}

Se captaron un total de 156 pacientes con cirrosis, de los cuales 25 desarrollaron IEC (16\%).

La distribución por género demostró un predominio del sexo masculino (68\%). La media de edad fue de 58 años (rango 16-73). La principal etiología de la cirrosis fue la enfermedad hepática por alcohol (17). Las restantes fueron: enfermedad de Wilson (1), Hígado Graso Metabólico (1), virus hepatitis $C^{(1)}$, colangitis esclerosante primaria ${ }^{(1)}$ y cirrosis criptogénica ${ }^{(4)}$.

En la tabla 1 se presentan la lista de los 25 pacientes con sus características epidemiológicas, severidad de cirrosis, tipo de infección y agente etiológico responsable de la misma.

\begin{tabular}{|c|c|c|c|c|c|c|c|c|c|}
\hline N & Género & Edad & Etiología & CTP & MELD & EC & MO & $\mathbf{S}$ & Origen \\
\hline 1 & $\mathrm{~F}$ & 32 & CG & B & 10 & PBE & - & - & C \\
\hline 2 & $\mathrm{~F}$ & 60 & CG & C & 19 & PBE & E Faecium & $\mathrm{s}$ & $\mathrm{N}$ \\
\hline 3 & $\mathrm{M}$ & 71 & $\mathrm{OH}$ & C & 19 & PBE & - & - & C \\
\hline 4 & $M$ & 41 & $\mathrm{OH}$ & C & 23 & PBE & E.Coli & $\mathrm{s}$ & $C$ \\
\hline & $\mathrm{F}$ & 54 & CG & C & 23 & PBE & E. Coli & BLEE & $\mathrm{N}$ \\
\hline 6 & $M$ & 64 & $\mathrm{OH}$ & C & 30 & $\mathrm{BE}$ & $\begin{array}{c}\text { K. } \\
\text { Pneumoníae }\end{array}$ & $S$ & C \\
\hline 7 & $\mathrm{~F}$ & 73 & $\mathrm{OH}$ & $C$ & 17 & $\mathrm{BE}$ & $\begin{array}{c}\mathrm{K} . \\
\text { Pneumoniae }\end{array}$ & BLEE & $\mathrm{N}$ \\
\hline 8 & $M$ & 72 & $\mathrm{OH}$ & $C$ & 15 & PBE & E. Coli & $S$ & C \\
\hline 9 & $\mathrm{~F}$ & 41 & EW & C & 35 & PBE & C. Albicans & - & $\mathrm{N}$ \\
\hline 10 & $M$ & 51 & $\mathrm{OH}$ & B & 10 & PBE & - & - & C \\
\hline 11 & $M$ & 72 & $\mathrm{OH}$ & C & 18 & PBE & E. Coli & $\mathrm{s}$ & C \\
\hline 12 & $\mathrm{~F}$ & 61 & CG & C & 20 & PBE & - & - & $C$ \\
\hline
\end{tabular}


Tabla 1: Lista de pacientes cirróticos hospitalizados que presentaron un IEC $(n=25)$

\begin{tabular}{|c|c|c|c|c|c|c|c|c|c|}
\hline 13 & $\mathrm{~F}$ & 70 & $\mathrm{OH}$ & $\mathrm{C}$ & 16 & $\mathrm{BE}$ & $\mathrm{E}$ Faecalis & $\mathrm{R}$ & $\mathrm{N}$ \\
\hline 14 & $\mathrm{M}$ & 38 & $\mathrm{OH}$ & $\mathrm{C}$ & 25 & $\mathrm{EBE}$ & - & - & $\mathrm{N}$ \\
\hline 15 & $\mathrm{M}$ & 16 & $\mathrm{CEP}$ & $\mathrm{C}$ & 16 & $\mathrm{PBE}$ & - & - & $\mathrm{C}$ \\
\hline 16 & $\mathrm{M}$ & 67 & $\mathrm{OH}$ & $\mathrm{B}$ & 14 & $\mathrm{BE}$ & $\begin{array}{c}\mathrm{K} . \\
\text { Pneumoniae }\end{array}$ & $\mathrm{S}$ & $\mathrm{N}$ \\
\hline & $\mathrm{M}$ & 48 & Viral & $\mathrm{A}$ & 10 & $\mathrm{BE}$ & $\mathrm{S}$. Agalacbae & $\mathrm{S}$ & $\mathrm{C}$ \\
\hline 18 & $\mathrm{M}$ & 63 & $\mathrm{OH}$ & $\mathrm{C}$ & 39 & $\mathrm{BE}$ & $\begin{array}{c}\mathrm{K} . \\
\text { Pneumoniae }\end{array}$ & $\mathrm{BLEE}$ & $\mathrm{N}$ \\
\hline 19 & $\mathrm{~F}$ & 74 & $\mathrm{NASH}$ & $\mathrm{B}$ & 8 & $\mathrm{PBE}$ & - & - & $\mathrm{C}$ \\
\hline 20 & $\mathrm{M}$ & 63 & $\mathrm{OH}$ & $\mathrm{B}$ & 14 & $\mathrm{BE}$ & $\mathrm{E}$. Coli & $\mathrm{S}$ & $\mathrm{RSS}$ \\
\hline 21 & $\mathrm{M}$ & 68 & $\mathrm{OH}$ & $\mathrm{C}$ & 25 & $\mathrm{BE}$ & $\mathrm{E}$ Cloacae & $\mathrm{R}$ & $\mathrm{N}$ \\
\hline 22 & $\mathrm{M}$ & 68 & $\mathrm{OH}$ & $\mathrm{B}$ & 23 & $\mathrm{BE}$ & $\mathrm{K}$. Oxytoca & $\mathrm{R}$ & $\mathrm{N}$ \\
\hline 23 & $\mathrm{M}$ & 68 & $\mathrm{OH}$ & $\mathrm{B}$ & 21 & $\mathrm{PBE}$ & - & - & $\mathrm{N}$ \\
\hline 24 & $\mathrm{M}$ & 53 & $\mathrm{OH}$ & $\mathrm{C}$ & 33 & $\mathrm{BE}$ & $\mathrm{E}$. Faecalis & $\mathrm{R}$ & $\mathrm{N}$ \\
\hline 25 & $\mathrm{M}$ & 71 & $\mathrm{OH}$ & $\mathrm{C}$ & 28 & $\mathrm{PBE}$ & $\begin{array}{c}\text { K. } \\
\text { Pneumoniae }\end{array}$ & $\mathrm{S}$ & $\mathrm{C}$ \\
\hline
\end{tabular}

Abreviaturas: $M$, masculino; $F$ femenino; $C G$, criptogénica; $\mathrm{OH}$, alcohólica; EW, enfermedad de Wilson; CEP, colangitis esclerosante primaria; NASH, esteatohepatitis no alcohólica; PBE, peritonitis bacteriana espontánea; EBE, empiema bacteriano espontáneo; BE, bacteriemia espontánea; S, sensible; R, resistente; C, comunitario; N, nosocomial; RSS, relacionada con los servicios de salud.

En cuanto a la severidad de la cirrosis, 24 de los 25 pacientes (96\%) se encontraban en un estadio avanzado de la enfermedad. Según score de Child-Pugh, 7 fueron estadio B y 17 estadio $\mathrm{C}$, mientras que el paciente restante se presentó con un score de Child-Pugh A La media de MELD-Na fue de 20 puntos (rango 10-39) (Gráfico 1).

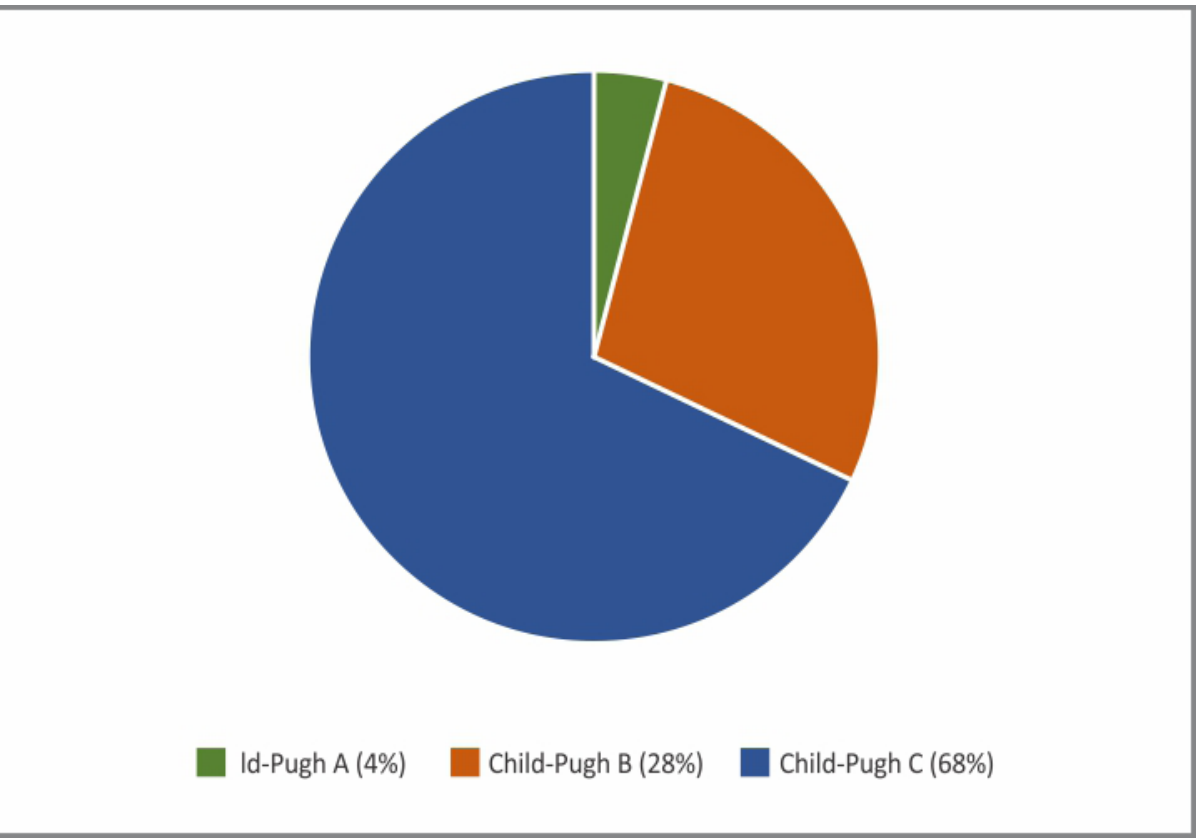

Del total de pacientes incluidos, 13 presentaron PBE, 10 BE y 2 EBE (Gráfico 2).

De las 13 PBE - 7 fueron a cultivo negativo, 3 a Escherichia coli (S), 1 a Escherichia coli BLEE, 1 a K. pneumoniae (S) y 1 a Candida albicans.

De las 10 BE - 4 fueron a Klebsiella pneumoniae, 2 (S) y 2 BLEE, 1 a E. coli (S), 2 a E. faecalis $(R), 1$ a K. oxytoca (R), 1 a S. agalactiae (S) y 1 a E. cloacae (R).

De las 2 EBE - 1 fue a cultivo negativo y 1 a Enterococcus faecium (S). 
Gráfico 2: Infecciones espontáneas en el cirrótico encontradas y sus características microbiológicas $(\mathrm{n}=25)$
Gráfico 3: Perfiles de resistencia en las infecciones espontáneas de cirróticos asociadas a la comunidad y nosocomiales $(n=25)$

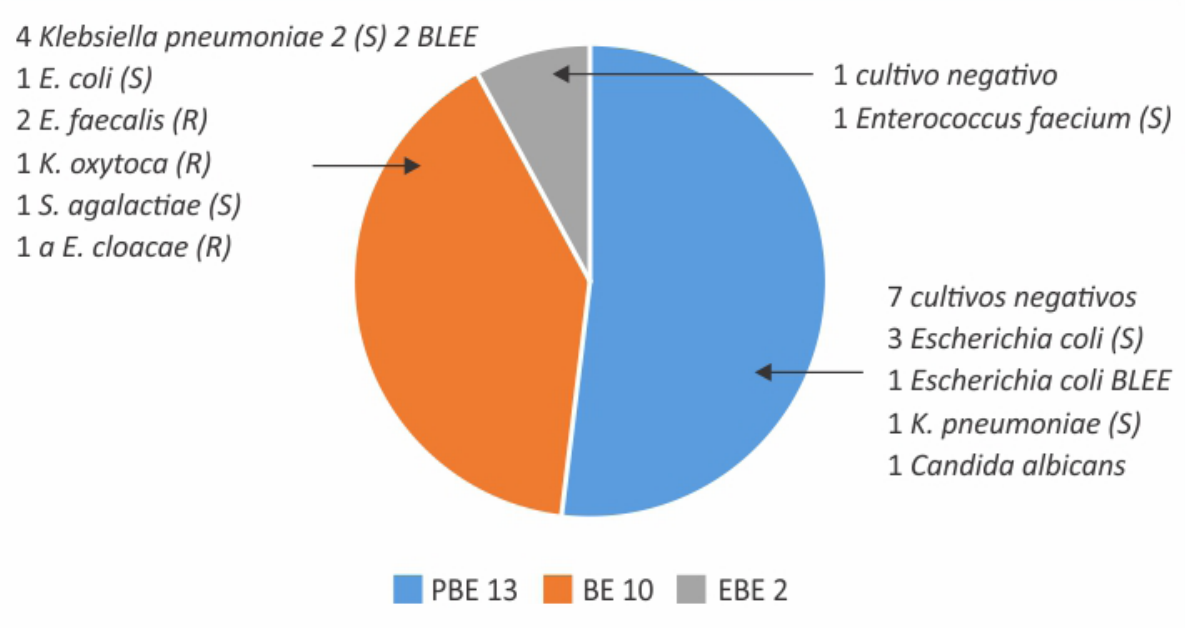

Abreviaturas: PBE, peritonitis bacteriana espontánea; BE, bacteriemia espontánea; EBE, empiema bacteriano espontáneo

En 12 casos las IEC fueron adquiridas en la comunidad, y en las restantes 13 la adquisición fue nosocomial.

De las 12 infecciones comunitarias, 6 fueron a cultivos negativos, 3 a Escherichia coli (S), 1 a S. agalactiae (S) y 2 a Klebsiella pneumoniae (S).

Las 13 infecciones nosocomiales fueron: 2 a cultivo negativo, 1 a Enterococcus faecium (S), 1 a K. pneumoniae (S), 1 a E. coli (S), 3 cepas productoras de BLEE (1 Escherichia coli y 2 Klebsiella pneumoniae), 2 a E. faecalis (R), 1 K. oxytoca (R), 1 E. cloacae (R) y 1 Candida albicans (Figura 3).

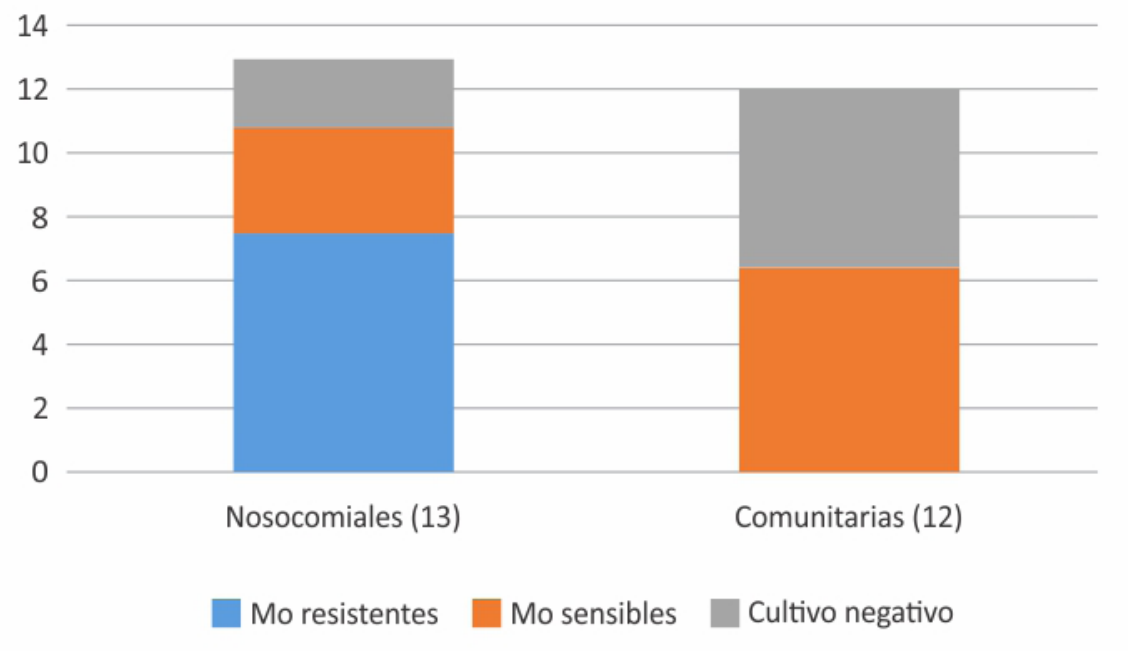

\section{Discusión}

Diferentes trabajos han demostrado que aproximadamente un $34 \%$ de los cirróticos internados presentan infecciones intercurrentes, de las que hasta un $38 \%$ serían IEC, representando aproximadamente un $13 \%$ del total.

Por razones de frecuencia, la principal es la PBE (66\%), seguida de las BE (32\%), y del EBE (2\%).

En el presente trabajo un 16\% de los pacientes cirróticos ingresados cursaron una IEC, ya sea al ingreso o durante la internación, siendo la PBE la más frecuente (52\%), seguida de las BE (40\%) y del EBE (8\%). 
Según surge de la bibliografía, este tipo de infecciones ocurren generalmente en pacientes con cirrosis avanzada, en esta serie la mayoría de los pacientes que desarrollaron una IEC correspondían a estadios B y C de Child-Pugh, con una media de MELD de 20 puntos.

Diversas publicaciones destacan que los microorganismos más frecuentemente aislados en las IEC corresponden a BGN. Los MOMR más frecuentes en el paciente cirrótico son las Enterobacterias productoras de betalactamasa de espectro extendido, (principalmente Escherichia coli y Klebsiella pneumoniae), seguido de Enterobacterias resistentes a carbapenemasas, Staphylococcus aureus meticilino resistente y Enterococcus vancomicina resistentes.

De los 25 pacientes que desarrollaron IEC en esta serie, fue posible el aislamiento microbiológico en 17 casos, mientras que en los restantes 8, correspondientes a PBE y EBE a cultivos negativos. En dichos casos el diagnóstico de la infección fue posible mediante el recuento de $\mathrm{PMN}$ en el líquido de ascitis o líquido pleural.

En 12 de los 17 pacientes con microorganismo identificado se aislaron BGN. Los 4 restantes fueron a Bacilos Gram Positivos (BGP) y Cándida (1). En cuanto a la sensibilidad antibiótica en este reporte se identificaron 3 cepas productoras de BLEE $(2 \mathrm{~K}$. pneumoniae, $1 \mathrm{E}$. coli), y 4 microorganismos con otros perfiles de resistencia ATB (2 E. faecalis, $1 \mathrm{~K}$. oxytoca, y $1 \mathrm{E}$. cloacae), todas en el grupo de IEC de adquisición nosocomial.

Del total de pacientes que cursaron una IEC con aislamiento microbiológico, se destaca una prevalencia de MOMR del $41 \%$. Además, las IEC por BGP y por Cándida albicans fueron en su mayoría adquiridas durante la internación, mientras que las IEC a cultivos negativos y aquellas a BGN sensibles fueron principalmente adquiridas en la comunidad.

La heterogeneidad de los patrones de resistencia en las diferentes regiones sugiere que las guías deberían adaptarse tomando en cuenta la epidemiología local, por medio de un abordaje multidisciplinario de la situación de estos pacientes.

\section{Conclusiones}

Los resultados obtenidos en el presente estudio coinciden con los reportes internacionales respecto a la prevalencia de IEC y las características microbiológicas de las mismas. Así mismo, es un hallazgo destacable el hecho de que la mayoría de las infecciones adquiridas en la comunidad fueron a cultivos negativos, o producidas por microorganismos sensibles, mientras que en las IEC nosocomiales la identificación del microorganismo responsable de la misma fue más frecuente y con mayores perfiles de resistencia antibiótica. Dado que el aislamiento de microorganismos y su perfil de sensibilidad y resistencia permite seleccionar la mejor estrategia antibiótica, el conocimiento de la epidemiología local es fundamental para instituir tratamientos empíricos ajustados a la realidad microbiológica de cada centro o región geográfica. Continuar esta línea de trabajo, extendiéndose a otras infecciones del cirrótico (NAC, ITU, infecciones de piel y partes blandas), e incrementando el número de centros participantes, podría ser de gran utilidad para cumplir este objetivo.

\section{Limitaciones del estudio:}

Además de las limitaciones inherentes al tipo de estudio (descriptivo y transversal) y al " $\mathrm{n}$ " pequeño de la muestra, cabe destacar que la recolección de datos fue realizada en base a la revisión de historias clínicas de pacientes asistidos por diferentes profesionales. Por dicha razón, y al no existir intervención alguna por parte de los autores, la actuación clínica no fue siguiendo protocolos prestablecidos, lo que de cierta manera no permite homogeneizar la actuación clínica. Por tanto, la solicitud de estudios (p.ej. reactantes de fase agua, cultivos microbiológicos), los tiempos en los que fueron solicitados, y los informes microbiológicos (p.ej. antibiogramas, perfiles de resistencia antibiótica) fueron diferentes entre ambas instituciones, incluso dentro de un mismo centro asistencial, dependiendo del equipo médico actuante.

\section{Conflicto de interés}

Los autores declaran no presentar conflictos de interés para el desarrollo de este estudio. 


\section{Bibliografía}

1- Fernández J. Bacterial infections in cirrhosis: Epidemiological changes with invasive procedures and norfloxacin prophylaxis. Hepatology. 2002;35:140- 148. doi: 10.1053/jhep.2002.30082.

2- Tandon P, Garcia-Tsao G. Bacterial infections, sepsis, and multiorgan failure in cirrhosis. Semin. Liver Dis. 2008;28:26-42. doi: 10.1055/s-2008-1040319.

3- Marciano S, Haddad L, Martínez AP, Posadas ML, Piñero F, Mora GJ, et al. Ultra-sensitive procalcitonin may help rule out bacterial infections in patients with cirrhosis. Ann. Hepatol. 2014;13:541-547. doi. org/10.1016/S1665-2681(19)31254-2.

4- Borzio M, Salerno F, Piantoni L, Cazzaniga M, Angeli P, Bissoli F, et al. Bacterial infection in patients with advanced cirrhosis: a multicentre prospective study. Dig. Liver Dis. 2001; 33: 41-48. doi: 10.1016/ s1590-8658(01)80134-1.

5- Foreman MG, Mannino DM, Moss M. Cirrhosis as a risk factor for sepsis and death: analysis of the National Hospital Discharge Survey. Chest. 2003; 124: 1016-1020. doi: 10.1378/chest.124.3.1016.

6- Terg R, Gadano A, Cartier M, Casciato P, Lucero R, Muñoz A, et al. Serum creatinine and bilirubin predict renal failure and mortality in patients with spontaneous bacterial peritonitis: a retrospective study. Liver Int. 2009;29:415-419. DOI: 10.1111/j.1478-3231.2008.01877.x.

7- Reuken PA, Stallmach A, Bruns T. Mortality after urinary tract infections in patients with advanced cirrhosis - Relevance of acute kidney injury and comorbidities. Liver Int. 2013;33:220-230. doi. org/10.1111/liv.12029.

8- Chen CH, Shih CM, Chou JW, Liu YH, Hang LW, Hsia TC, et al. Outcome predictors of cirrhotic patients with spontaneous bacterial empyema. Liver Int. 2011;31:417-424. doi: 10.1111/j.14783231.2010.02447.x.

9- Marciano S, Dirchwolf M, Bermudez CS, Sobenko N, Haddad L, Genre Bert F, et al. Spontaneous bacteremia and spontaneous bacterial peritonitis share similar prognosis in patients with cirrhosis: a cohort study. Hepatology. Int. 2018;12:181-190. 20 doi: 10.1007/s12072-017-9837-7.

10- Marciano S, Valverde M, Dirchwolf M, Gutierrez-Acevedo MN, Gadano A. The Importance of Knowing the Local Epidemiology When a Patient With Cirrhosis Acquires a Bacterial Infection. Clin Liver Dis. 2020; 16(3): 87-90

11- Arvaniti V, D'Amico G, Fede G, Manousou P, Tsochatzis E, Pleguezuelo M, et al. Infections in patients with cirrhosis increase mortality four-fold and should be used in determining prognosis. Gastroenterology. 2010;139:1246-56, 1256.e1-5. doi: 10.1053/j.gastro.2010.06.019.

12- Gustot T, Durand F, Lebrec D, Vincent J-L, Moreau R. Severe sepsis in cirrhosis. Hepatology. 2009;50:2022-2033. doi: 10.1002/hep.23264.

13- Angeli P, Bernardi M, Villanueva C, Francoz C, Mookerjee RP, Trebicka J, et al. EASL Clinical Practice Guidelines for the management of patients with decompensated cirrhosis. J Hepatol [Internet]. 2018;69(2):406-60. Available from: https://linkinghub.elsevier.com/retrieve/pii/S0168827818319664.

14- Wiest R, Lawson M, Geuking M. Pathological bacterial translocation in liver cirrhosis. J Hepatol. 2014; 60:197-209. doi: 10.1016/j.jhep.2013.07.044.

15- Albillos A, Lario M, Álvarez-Mon M. Cirrhosis-associated immune dysfunction: distinctive features and clinical relevance. J Hepatol. 2014;61: 1385-1396. doi: 10.1016/j.jhep.2014.08.010.

16- Fernández J, Acevedo J, Castro M, Garcia O, de Lope CR, Roca D, et al. Prevalence and risk factors of infections by multiresistant bacteria in cirrhosis: a prospective study. Hepatology. 2012; 55:1551-1561. doi: 10.1002/hep.25532

17- Piroth L, Pechinot A, Di Martino V, Hansmann Y, Putot A, Patry I, et al. Evolving epidemiology and antimicrobial resistance in spontaneous bacterial peritonitis: a two-year observational study. BMC Infect. Dis. 2014; 14:287. doi: 10.1186/1471-2334-14-287.

18- Ginès P, Angeli P, Lenz K, Møller S, Moore K, Moreau R. EASL clinical practice guidelines on the management of ascites, spontaneous bacterial peritonitis, and hepatorenal syndrome in cirrhosis. J. Hepatol. 2010; 53: 397-417. DOI: 10.1016/j.jhep.2010.05.004

19- Jalan R, Fernandez J, Wiest R, Schnabl B, Moreau R, Angeli P, et al. Bacterial infections in cirrhosis: a position statement based on the EASL Special Conference 2013. J. Hepatol. 2014; 60: 1310-1324. 11 doi: 10.1016/j.jhep.2014.01.024.

20- Piano S, Brocca A, Mareso S, Angeli P. Infections complicating cirrhosis. Liver Int. 2018; 38 Suppl 1:126-133. doi: 10.1111/liv.13645. 


\section{Aporte cada autor al trabajo}

Martín Elizondo: Concepción y diseño del trabajo, recolección de datos, análisis e interpretación de los resultados y redacción del manuscrito.

Bruno Mirza: Concepción y diseño del trabajo, recolección de datos, análisis e interpretación de los resultados y redacción del manuscrito.

María Noel García: Análisis e interpretación de los resultados y redacción del manuscrito.

Marcelo Valverde: Concepción y diseño del trabajo, análisis e interpretación de los resultados, redacción del manuscrito y revisión crítica del manuscrito.

Solange Gerona: Concepción y diseño del trabajo y revisión crítica del manuscrito.

Dra. Gabriela Ormaechea: Concepción y diseño del trabajo y redacción del manuscrito. 